\title{
CENTROS/CONJUNTOS HISTÓRICOS DE CANARIAS: IDENTIDAD EN UN TERRITORIO ISLEÑO. TRAYECTORIAS 1973-2018
}

\author{
Juan Sebastián López García \\ Universidad de Las Palmas de Gran Canaria
}

\section{RESUMEN}

Los conjuntos históricos de Canarias concentran una parte significativa del patrimonio isleńo y algunos por su carácter emblemático son iconos de la identidad canaria, especialmente los rectores del territorio en los sistemas urbanos de cada isla. La valoración de estos núcleos y su conversión en bienes de interés cultural, categoría conjunto histórico, comenzó en 1973 con la declaración del barrio de Vegueta, Las Palmas de Gran Canaria, y alcanzó su marca Unesco con la categoría de Patrimonio de la Humanidad a favor de La Laguna en 1999. Este proceso cuenta con tres marcos competenciales diferentes: Gobierno central, comunidad autónoma y cabildos insulares. Las declaraciones se ajustan a la legislación del momento y en paralelo se suman otros factores, donde se aprecian planteamientos diferentes entre provincias e islas.

Palabras Clave: Canarias, centro histórico, conjunto histórico, territorio, identidad cultural.

\section{CENTERS/HISTORICAL SETS OF THE CANARY ISLANDS: IDENTITY IN AN ISLEÑO TERRITORY. TRAJECTORIES 1973-2018}

Abstract

The historical sites of the Canary Islands concentrate a significant part of the island's heritage and some of them, because of their emblematic carachter, are icons of the Canarian identity, especially those that play a large role in shaping the territorial structure of the urban systems of each island. The recognition of these historic centers and their conversion into "Bienes the interés cultural» (goods of cultural interest) within the category of «Conjunto histórico), began in 1973 with the declaration of the "Barrio de Vegueta" (Las Palmas de Gran Canaria), and reached its Unesco standing with the category of World Heritage in favor of La Laguna in 1999. This process has three different competence frameworks: central government, autonomous community government, and island councils. The declarations are adjusted to the legislation of the moment and in parallel other factors are added, if and when different approaches between provinces and islands are appreciated.

KeYwords: Canary Islands, historic center, historical site, territory, cultural identity. 


\section{INTRODUCCIÓN}

La valoración de los centros históricos de Canarias y su conversión en bienes de interés cultural (BIC), categoría conjunto histórico, comenzó hace más de cuatro décadas con la declaración del barrio de Vegueta, Las Palmas de Gran Canaria, en 1973 y alcanzó su marca de calidad internacional con la inclusión de San Cristóbal de La Laguna en la lista de Patrimonio de la Humanidad de la Unesco en 1999. En ese proceso de más de cuarenta años se ha asistido a tres marcos legislativos diferentes y ha cambiado el ámbito competencial, de tal manera que desde el Gobierno central se ha pasado a la comunidad autónoma canaria, organismo que además traspasó algunos servicios y funciones de los mismos a los cabildos de cada isla para los bienes ubicados en su ámbito. Así, en las declaraciones (los primeros como «conjunto histórico-artístico», y los siguientes BIC «conjunto histórico», según la legislación de cada momento) hay decretos y reales decretos publicados a nivel nacional en el $B O E$, mientras que a partir de 1985 serán decretos del Gobierno de Canarias (unos pocos como Orden).

A estos antecedentes se suman otros factores, donde se aprecian planteamientos diferentes entre las dos provincias (Las Palmas y Santa Cruz de Tenerife) y además entre islas, de tal manera que actualmente la mayoría de los conjuntos históricos están ubicados en Tenerife, seguida de Gran Canaria, dos en La Palma y uno en Fuerteventura y Lanzarote respectivamente, estando ausentes La Gomera y El Hierro. Sin embargo, este resultado no ha seguido una trayectoria regular, sino que se inserta en distintas etapas, que han ido marcando diferentes tendencias, tales como el inicio en 1993 de las declaraciones de conjuntos de arquitectura rural, la presencia de varios discontinuos en una misma ciudad, la existencia de diversos en un municipio, etc.

Los conjuntos históricos de Canarias concentran una parte muy significativa del patrimonio de las islas, pero también muchos de ellos por su carácter emblemático son iconos de la identidad isleña, especialmente los que han sido rectores de los sistemas urbanos de cada isla, matrices de ciudades y villas, con la peculiaridad de que algunos datan de la época prehispánica. Muchas de las islas reflejan la "construcción histórica» de su territorio de forma muy clara a partir de sus conjuntos históricos, lectura que se aprecia especialmente en Gran Canaria, La Palma, Lanzarote y Fuerteventura, y en menor medida en Tenerife.

Frente a conjuntos históricos bastante homogéneos, otros ofrecen un patrimonio más diverso, estando presentes en ambos casos muestras muy destacadas de lo tangible e intangible. Estos bienes son espacio preferente de la cultura y el arte (incluido el efímero) y en su valoración actual muchos de ellos se han convertido en un magnífico recurso para el turismo, sobre todo para comarcas que no coinciden con las zonas turísticas de sol y playa, circunstancia muy adecuada como complemento a las economías locales y como oferta para unas islas que tienen su principal fuente económica en la llegada de visitantes.

Una parte muy destacada del patrimonio cultural de Canarias se concentra en sus centros históricos, reflejo del proceso de su sistema urbano desde el período anterior a la conquista, de ahí que algunos de ellos tengan una significación muy 
especial. El marco insular, aparte de algunas ciudades que han estado presentes en la escena regional, hacen que el primer referente sea la propia isla en una valoración de lo que cada centro histórico le aporta.

El presente artículo revisa trabajos anteriores e incluye el análisis hasta el año 2018, de tal manera que muestra de forma mucho más completa la trayectoria seguida por los centros y conjuntos históricos de Canarias en las últimas décadas, completando y ofreciendo relecturas actualizadas de los estudios anteriores, ya que como podrá apreciarse, la situación ha variado bastante en muchos de los aspectos y, en general, ha aumentado la capacidad de convertirlos en un recurso tanto para la identidad canaria como para una oferta cultural de calidad para el turismo. En muchos casos el fenómeno queda ligado a un paisaje cultural, localizable preferentemente en áreas rurales donde la interacción humana con la naturaleza ha creado espacios singulares con diversificación de aportes tangibles e intangibles a lo largo de los siglos, que necesariamente deben ser considerados recursos sostenibles, por su vulnerabilidad y riesgo de pérdida irreversible, incluso muchos de ellos actualmente abandonados y en zonas casi despobladas. La particularidad de cada isla y de cada lugar está permitiendo establecer itinerarios de patrimonio diverso, adecuados a su ámbito y que por tanto pueden ser competitivos al diferenciarse por su singularidad ${ }^{1}$.

\section{UN PROCESO ASIMÉTRICO INTERINSULAR}

\section{Aspectos Generales}

La trayectoria de los centros históricos de Canarias ha sido diversa en las distintas etapas en las que se puede dividir su proceso de valoración. En una primera aproximación al tema se podrían seleccionar tres ítems correspondientes a fechas clave de estos bienes inmuebles canarios:

1973. La declaración del primer conjunto histórico artístico, según la denominación de entonces: barrio de Vegueta, Las Palmas de Gran Canaria.

1999. La declaración del primer conjunto canario como Patrimonio de la Humanidad. San Cristóbal de La Laguna.

2018. La inclusión del conjunto histórico de arquitectura popular en casacueva Barranco Hondo de Abajo (Gáldar) en la candidatura española a Patrimonio Mundial de «Risco Caído y los espacios sagrados de montaña de Gran Canaria (Santuario de Risco Chapín, Sierra del Bentayga, Mesa de Acusa y Risco Caído)» (Agaete, Artenara, Gáldar y Tejeda).

${ }^{1}$ Para la visión general de los conjuntos y centros históricos del archipiélago, vid. López García, Juan Sebastián (2010): Los centros históricos de Canarias, Las Palmas de Gran Canaria. 
Estos hitos forman parte de un historial más complejo, asimétrico entre islas, que se ha ido constituyendo en más de cuarenta años, ya que en 2023 se cumple el medio siglo del primer BIC categoría conjunto histórico, según la terminología actual de las leyes española de 1985 y canaria ${ }^{2}$, declarados los primeros por la normativa anterior correspondiente a la Ley de $1933^{3}$. Es decir, que en esta trayectoria concurren tres marcos legislativos, correspondiendo el primero con una Ley de la II República, que estuvo vigente durante el régimen republicano, franquista, transición y democracia, con ámbito nacional, y la ley canaria, en virtud de transferencias en materia de cultura a la comunidad autónoma. El panorama se completa con el traspaso de funciones y servicios a los cabildos, que ha permitido llevar la gestión del patrimonio a cada una de las siete corporaciones insulares, incluida la potestad de la incoación de los bienes de interés cultural en su ámbito insular $r^{4}$.

Como se dijo, la valoración de los conjuntos históricos de Canarias comenzó en 1973. Antes de esa fecha se ha considerado que en las islas «se vivía una falta de interés por sus centros urbanos antiguos, que se ponía de manifiesto en las actuaciones de las distintas administraciones... En la misma Universidad no era tema de estudio... El desconocimiento de la Historia y de la Ciudad fueron dos componentes que informaron toda una política y una actitud ante los centros urbanos antiguos canarios" ${ }^{5}$. Todo esto en un ambiente de las décadas de los sesenta y comienzos de los setenta, bajo el signo del desarrollismo y al margen de la preocupación por la ciudad histórica y un debate sobre la misma. A principios de los setenta se comienza a notar un cambio de tendencia en cuanto a los estudios e investigaciones que empiezan a destacar en el departamento de Historia del Arte de la Universidad de La Laguna y en la Escuela de Arquitectura de Las Palmas ${ }^{6}$.

Los conjuntos históricos pioneros se declararon en la etapa 1973-1982, fruto de las incoaciones tramitadas por las desaparecidas delegaciones provinciales de Bellas Artes que existieron hasta las transferencias en materia de cultura y patrimonio histórico a la comunidad autónoma. Aparte del primero de los declarados en 1973, en los correspondientes a la provincia de Las Palmas entre 1978 y 1982 se cuentan siete conjuntos de ellos. Estos primeros once bienes fueron: barrio de Vegueta, Las Palmas de Gran Canaria (1973); Santa Cruz de La Palma (1975); La Orotava, Tene-

${ }^{2}$ Ley 16/1985, de 25 de junio, del Patrimonio Histórico Español, BOE n. ${ }^{\circ}$ 155, de 29 de junio; corrección de errores en $B O E$ n. ${ }^{\circ} 296$, de 11 de diciembre de 1985). Ley 4/1999, de 15 de marzo, de Patrimonio Histórico de Canarias, BOC n. ${ }^{\circ}$ 36, de 24 de marzo de 1999.

${ }^{3}$ Ley sobre Defensa, Conservación y Acrecentamiento del Patrimonio Histórico Nacional de 13 de mayo de 1933 (Gaceta de Madrid, año CCLXXII, tomo II, n. ${ }^{\circ}$ 145, 25 de mayo de 1933).

${ }^{4}$ Decreto 152/1994, de 21 de julio, de transferencias de funciones de la Administración Pública de la Comunidad Autónoma de Canarias a los Cabildos Insulares en materia de cultura, deportes y patrimonio histórico-artístico, BOC 92, de 28 de julio de 1994.

5 López García, Juan Sebastián (1989): «Etapas para un estudio reciente de los centros históricos de Canarias», en Arquitectura y Urbanismo en Canarias 1968-1988, Las Palmas de Gran Canaria, pp. 265-266.

"Fraga González, Carmen (1985): «Arte en Canarias: estado de la cuestión», en V Coloquio de Historia Canario-Americana (1982), Las Palmas de Gran Canaria, III, pp. 35-65. 
rife (1976); Betancuria, Fuerteventura (1978); Teror, Gran Canaria (1979), Arucas, Gran Canaria (1979); Tacoronte, Tenerife (1980); Teguise, Lanzarote (1980); plaza de Santiago, Gáldar, Gran Canaria (1981), San Juan y San Francisco, Telde, Gran Canaria (1981); Santa María de Guía, Gran Canaria (1982)7.

Al cierre de esta primera etapa con los declarados por el Gobierno central, se aprecian algunas características: «De una lectura rápida de esta nómina de nombres y fechas se ve la casi paridad de declaraciones en las décadas de los setenta y los ochenta, con la peculiaridad que en un segmento de nueve ańos (1973-1982) se declaran una gran mayoría. También se puede apreciar un cierto desajuste interinsular, ya que siete pertenecen a Gran Canaria, cinco a Tenerife, uno a La Palma, Lanzarote y Fuerteventura, respectivamente. Todo esto no es consecuencia sino del hecho de tratarse de una proceso abierto, todavía con grandes posibilidades de cambios... ${ }^{8}$. Este texto es de 1997, con más de veinte años, el cual es muy interesante para cotejar con la situación actual, porque ya en esa fecha se recalcaba que las probabilidades de cambios eran muy grandes y así ha sido. Todos esos bienes pioneros fueron declarados por el Gobierno central y promovidos por las entonces delegaciones provinciales de Bellas Artes de Las Palmas y Santa Cruz de Tenerife, desde donde se iniciaron los trámites.

Con las competencias transferidas en materia de cultura en $1982^{9}$, el Gobierno autónomo decreta la declaración a favor de San Cristóbal de La Laguna en 1985, a la que siguieron muchos más, de tal manera que dos pertenecen a los años ochenta del siglo pasado (los cinco de principios de esa década fueron declarados por el Gobierno central, tal como se ha visto), cinco en los noventa, dieciocho entre 2001-2009 y cinco a partir del año 2010. De esta etapa declaratoria autonómica de conjuntos históricos destaca como una de las novedades la incorporación de los de carácter rural y de arquitectura popular, grupo que inició el grancanario Barranco Hondo de Abajo (Gáldar) en 1993; también la inclusión de algunos bienes que se acogieron a esta categoría pero que posiblemente se ajusten mejor a otras figuras, por lo menos desde un punto de vista académico y no político, es decir, que en un estricto tratamiento de centros o conjuntos históricos no se les hubiera considerado como tales, caso de la Mayordomía de Tamaraceite (Las Palmas de Gran Canaria ${ }^{10}$. También se incoó la modificación del perímetro del casco histórico de Santa María de Guía, etc. Muy importante fue que en estos años, en paralelo, se

7 No consideramos en esta lista a Las Raíces, La Esperanza, El Rosario, Tenerife (1964) y el castillo de San Gabriel, su camino de acceso y su puente levadizo, Arrecife, Lanzarote (1979), por entender que es discutible para ellos esa figura y que se ajustarían mejor a otra categoría de bien de interés cultural.

${ }^{8}$ López García, Juan Sebastián (1997): «La ciudad histórica en Canarias: patrimonio cultural y territorio", Mérida. Ciudad y Territorio. Revista Científica, n. ${ }^{\circ}$ 1, pp. 174-175.

${ }^{9}$ R.D. 27/1982, de 12 de agosto, sobre transferencia de competencias, funciones y servicios de la Administración del Estado a la Junta de Canarias en materia de Cultura BOE. 05-11-1982. Correcciones errores BOE 30-11-1985.

${ }^{10}$ Posiblemente estaría más ajustado a la categoría monumento, con una delimitación que incluya las distintas construcciones. 
produjo la inscripción por parte de la Unesco del conjunto histórico de San Cristóbal de La Laguna como Patrimonio de la Humanidad en 1999. La ciudad de los adelantados fue la única que hizo una apuesta firme y decidida por este tema, aunque se planteó de forma más o menos diferente, unas con más empeño que otras, para Teguise, Betancuria, Las Palmas de Gran Canaria o Santa Cruz de La Palma, sin llegarse a formalizar en ninguno de los casos. De cualquier manera se entiende que actualmente hay pocas posibilidades para ese tipo de reconocimiento y la candidatura de Risco Caído (Agaete, Artenara, Gáldar y Tejeda), si bien integra un conjunto histórico de carácter rural, se hace en el ámbito del paisaje cultural. Por otra parte, la capital palmera ha reorientado su propuesta hacia el patrimonio inmaterial tomando como centro la Bajada de la Virgen de las Nieves, donde la ciudad histórica es el escenario cada lustro de una fiesta singular del Barroco con aportes posteriores.

En un repaso por islas, después de 1985 la tendencia más destacada ha sido el notable aumento de conjuntos históricos tinerfeños, isla donde falta alguno significativo. La Palma ha sumado uno más, Fuerteventura y Lanzarote continúan en la misma situación de uno en cada isla, pertenecientes a la etapa anterior, y siguen sin bienes de esta categoría La Gomera y El Hierro. En esta última etapa también hay que señalar que no se resolvieron favorablemente las propuestas de varios centros históricos como el tinerfeño de la villa de Candelaria (Tenerife) y el lanzaroteño lugar de Haría (Lanzarote). Ambos expedientes fueron cancelados y quedaron sin las medidas protectoras cautelares de las que disfrutaban.

La situación de mayor coherencia del panorama de conjuntos históricos declarados la ofrece la provincia de Las Palmas, aun analizando por separado las islas de Fuerteventura, Gran Canaria y Lanzarote (La Graciosa no cuenta con ninguno de ellos). Más contrastada es la provincia de Santa Cruz de Tenerife, con un panorama complejo en Tenerife, donde se localizan actualmente la mayoría de los conjuntos históricos de Canarias, comparando con los dos de La Palma y ninguno en La Gomera y El Hierro.

\section{FuerteVentura y LANZARote}

Empezando por las dos islas más orientales, ambas tienen declaradas sus antiguas cabeceras insulares, que lo fueron desde el siglo xv al xIx, Betancuria (BIC 1978) y Teguise (BIC 1980). En Lanzarote además se incoó Haría, el segundo núcleo que destacó en el territorio lanzaroteño como primer curato segregado de Teguise, pero después de varios años el expediente no prosperó. En cuanto a Arrecife, con los perímetros de bienes declarados y cercanos entre sí, tales como el castillo de San Gabriel (1979), iglesia de San Ginés (1990), Casa de los Arroyo (1990) y antigua sede del Cabildo Insular (2004) se puede crear un "conjunto histórico», categoría que sin embargo se utilizó para el referido castillo con el puente de las Bolas, que no responde bien a su carácter de monumento. Fuerteventura no ha tenido ninguna otra incoación de este carácter, aunque cuenta con alguna iniciativa ciudadana de propuesta para la posible declaración de BIC para Puerto del Rosario, que hereda por cambio de denominación al topónimo antiguo de Puerto de Cabras, que 
como núcleo se desarrolló a partir del siglo xIx y con jurisdicción hijuela del desaparecido municipio de Tetir. Esta isla presenta una especial dificultad de delimitación de sus bienes por su peculiar "forma urbana» anterior al siglo XIX, a causa de la dispersión y falta de colmatación de sus núcleos habitados. En síntesis, en cuanto a estas dos islas, sin ninguna aportación posterior a 1980, se puede considerar que poseen una situación adecuada, porque desde hace casi cuarenta años sus dos antiguas capitales son conjuntos históricos, las cuales poseen el más destacado patrimonio de cada una ellas, en cuanto a urbanismo, arquitectura, bienes muebles, documentales e intangibles.

\section{Gran Canaria}

El primer bien de este tipo declarado en Canarias fue el barrio de Vegueta, Las Palmas de Gran Canaria, en 1973, al que se unió más tarde el barrio de Triana (1990, publicado en 1993). Como se comentó, en muy pocos años se completó la primera fase del panorama insular con Teror (1979), Arucas (1979), Gáldar (1981), Telde (1981) y Santa María de Guía (1982), con lo que en menos de diez años quedaron declarados los cascos urbanos antiguos de las cinco ciudades de la isla, más una de sus villas. En los años noventa se declaró Barranco Hondo de Abajo (Gáldar), primer conjunto de arquitectura popular, en concreto de casacueva, declarado en Canarias en 1993. En 2010 se produjo la declaración a favor de la villa de Santa Brígida y en 2018 la calle Perojo, Las Palmas de Gran Canaria ${ }^{11}$. Hay que resaltar que en 1995 se declaró como conjunto histórico las Casas de la Mayordomía y ermita de San Antonio Abad, Tamaraceite, Las Palmas de Gran Canaria, que posiblemente hubiera quedado mejor acomodada en otra categoría. No tomando en consideración este último bien cultural, la isla contaría con diez conjuntos históricos, tanto urbanos como rurales, además destacándose que dentro de la ciudad de Las Palmas hay tres de ellos (Vegueta, Triana y Perojo) y dos en el municipio de Gáldar (centro ciudad y aldea de Barranco Hondo de Abajo). Por ubicaciones, la mayoría están en el norte, emplazados desde la costa hasta la cumbre, pasando por las medianías. Futuras declaraciones podrían engrosar este registro grancanario, aunque actualmente ofrece entre sus bienes la mejor representación de la isla, muy completo en lo territorial, y muy representativo en el ámbito del patrimonio arquitectónico y urbano (con sus bienes muebles e intangibles vinculados), en cuanto a lo erudito y lo popular, con algunas de las muestras singulares más importantes de Canarias. Entre los expedientes incoados se encuentran los conjuntos históricos de la villa de Agüimes (1991) y el caserío de Temisas (Agüimes, 1990) ${ }^{12}$.

${ }^{11}$ En cuanto al estado de los que cuentan con planes especiales vid. Lozano Mas, María Yasmina (2016): La planificación de los conjuntos históricos de Gran Canaria. Perspectiva critica desde su ordenación urbanistica, tesis doctoral, Universidad de Las Palmas de Gran Canaria.

12 Villa de Agüimes: R. de 8 de octubre de 1991. Caserío de Temisas, Agüimes, R. de 18 de diciembre de 1990 . 
El primer conjunto histórico declarado en Tenerife fue la villa de La Orotava, en 1976, siendo el segundo de la provincia santacrucera (recordemos que el primero fue Santa Cruz de La Palma, 1975), al que siguió Tacoronte en 1980, ambos declarados por el Gobierno de España. Los siguientes lo fueron por decretos del Gobierno de Canarias, iniciándose una tendencia que ha sido dominante en la comunidad autónoma canaria a partir de mediados de los ańos ochenta del siglo pasado, caracterizada por la presencia mayoritaria de declaraciones de conjuntos históricos tinerfeños, lo que en parte es lógico por los pocos que habían sido reconocidos con anterioridad. La lista autonómica se inicia con la ciudad de San Cristóbal de La Laguna (1985, publicado en BOC en 1986), con el puesto número doce de los canarios (desconsiderando Las Raíces, 1964, y el arrecifeño castillo de San Gabriel, 1979) y subsanando una circunstancia tan particular como que un centro histórico tan importante para las islas no tuviera esa categoría, lo que resultaba inexplicable. Como sabemos, catorce años después logra su reconocimiento de Patrimonio de la Humanidad. Seguida es la declaración de Tegueste en 1986, teniendo que pasar ocho ańos para publicarse el único de los ańos noventa, que fue la villa y puerto de Garachico (1994). La primera década del siglo Xxi ha sido la más fecunda para el reconocimiento de los conjuntos históricos tinerfeños, en cantidad y variedad, destacándose la presencia de núcleos sureños, ya que hasta ese momento todos los declarados estaban localizados en la banda norte. La relación comienza con Güímar (2001), Casas Altas en Jama, Arona y Vilaflor (2004), Masca, Buenavista del Norte (2004), Los Silos (2004), Icod de los Vinos (2005), villa de Arico (2005), Buenavista del Norte (2005), Icor, Arico (2005), Taucho, Adeje (2005), Realejo Bajo, Los Realejos (2005), Puerto de la Cruz ((2006), barrio del Toscal, Santa Cruz de Tenerife (2007), Arico el Nuevo, Arico (2007), barrios de los Hoteles-Pino de Oro, Santa Cruz de Tenerife (2007), Arona (2007), Antiguo Santa Cruz, Santa Cruz de Tenerife (2007), caseríos de Chirche y Aripe, Guía de Isora (2008) y Guía de Isora (2009). Estos conjuntos declarados en Tenerife (2001-2009) aportan novedades en la trayectoria declaratoria de la isla y por ende de Canarias. Por reparto geográfico, hay nueve tanto en el norte como en el sur, donde por primera vez aparecen en las listas tinerfeñas los de carácter rural, en número de cinco a partir de 2004 (once años después de que se declarara el primero de este tipo en Canarias: Barranco Hondo de Abajo, Gáldar, 1993), pertenecientes a seis municipios diferentes, ya que uno es compartido por dos jurisdicciones. Destaca la presencia de Santa Cruz de Tenerife, que en 2007 consigue la declaración de tres conjuntos históricos de diferente carácter, en tres barrios de la ciudad.

La declaración de dieciocho conjuntos históricos en una década para una misma isla no se había producido en Canarias, ni tan siquiera para toda la comunidad autónoma. Con posterioridad, en 2013 se declara San Miguel de Abona y en 2014 Realejo Alto, Los Realejos. Con estas declaraciones de principios del siglo XXI una serie de municipios tinerfeños pasaron a tener varios conjuntos históricos: Arico con tres (villa de Arico, 2005; Icor, 2005; y Arico el Nuevo, 2007), Santa Cruz de Tenerife también con tres (barrios de El Toscal, Los Hoteles-Pino de Oro y el 
Antiguo Santa Cruz, todos de 2007), con dos Buenavista del Norte (Masca, 2004; Buenavista del Norte, 2005), Guía de Isora (Chirche y Aripe, 2008; Guía de Isora, 2009), Arona (Casas Altas en Jama, compartido con Vilaflor; 2004) y Los Realejos (Realejo Bajo, 2005; Realejo Alto, 2014). Algunos de los referidos tuvieron largos procesos, incluso con una manifiesta oposición de los vecinos, como ocurrió en Icod de los Vinos, donde las alegaciones contrarias fueron numerosas; en el caso de Güímar se retrasó la declaración por modificación del perímetro de delimitación. Con respecto a esta isla, los cambios que se habían operado fueron más bien un reajuste entre el número de centros históricos que existían y los pocos que habían sido registrados como bienes de interés cultural, lo que se evidenciaba en este texto escrito en 1997: «El panorama de Tenerife se tendría que valorar en cuanto a sus centros históricos, es decir a los que tienen la potencialidad de ser declarados, ya que el número de los reconocidos es realmente bajo y no da idea de la importancia de los núcleos de la isla» ${ }^{13}$.

\section{La Palma}

La Palma cuenta con una de las declaraciones pioneras de la comunidad autónoma, ya que Santa Cruz de La Palma es de 1975, solo dos años después de producirse la laspalmeña del barrio de Vegueta, siendo ambos los únicos anteriores a la transición democrática. Es el más antiguo de la provincia de Santa Cruz de Tenerife, el ejemplo patrimonial de más valor y representatividad de la isla y uno de los destacados de Canarias. Cuarenta años después se le sumó la declaración de la villa de San Andrés (municipio de San Andrés y Sauces) en 2015. Ambos son muy notables en La Palma, aunque se puede completar con otros, como sería el caso de la ciudad de Los Llanos de Aridane.

\section{APORTES A LA IDENTIDAD: LECTURA TERRITORIAL Y VALOR CULTURAL}

Las declaraciones de los conjuntos históricos se han realizado sin ningún tipo de planificación, ni jerarquización; un ejemplo lo constituye San Cristóbal de La Laguna, que a pesar de su siempre reconocida importancia histórica, le anteceden varios bienes de interés cultural entre los que se encuentran algunos muy modestos y sin embargo es el único de Canarias que hasta el momento es Patrimonio de la Humanidad. Bien es cierto que en la provincia de Las Palmas pareciera que hubo un plan previo porque en una primera etapa se cerró un proceso con los más repre-

13 Vid. López García, Juan Sebastián (1997): «Ciudades históricas canarias. La pervivencia y la restauración del territorio", en Congreso Ciudades Históricas Vivas. Ciudades del pasado: pervivencia y desarrollo, tomo I, Mérida, p. 260. 
sentativos, pero posiblemente se deba al buen hacer y entendimiento del entonces director provincial de Bellas Artes, D. José Miguel Alzola, que además fue director de El Museo Canario, que si no marcó una estrategia con su actuación logró los mejores resultados.

Vistos actualmente en un mismo plano es muy difícil discernir sobre una escala de «valores emblemáticos y simbólicos» en los conjuntos históricos, ya que los reconocimientos se han hecho por los bienes más aparentes y no por sus significados no visibles. El carácter significativo como valor añadido no se ha tenido mucho en cuenta y se entiende que en algunos casos constituye un plus diferenciador y se podría decir que hasta de «alta calidad» del conjunto histórico ${ }^{14}$. Por si sirve de referencia, en un trabajo (2012) sobre las ciudades de La Antigua Guatemala (Sacatepéquez, Guatemala) y Guadalajara (Jalisco, México), ambas con bienes inmuebles Patrimonio de la Humanidad, se hizo una lectura de «revaloración emblemática», considerando en especial a sus plazas mayores como espacios de singularidad urbana, arquitectónica, cultural y territorial, donde junto a sus «formas artísticas» subyacen las funciones históricas intangibles de alcance territorial supranacional, para toda Centroamérica la primera y para la segunda el centro y norte de México con el amplio territorio de los Estados Unidos que perteneció a su audiencia y formó parte del Imperio español. Con anterioridad, en 1997 se había establecido algo para Canarias en la significación de sus centros históricos, donde se tendría en consideración que además de sus aportes culturales hubieran tenido un papel destacado en el origen del sistema urbano de las islas, con dos consideraciones interesantes que no eran excluyentes: origen prehispánico y matrices de otras ciudades y villas ${ }^{15}$.

Los conjuntos históricos son integrantes de la ciudad y pueden tener por su significación un notable vínculo territorial, de distinto alcance según los casos. $\mathrm{Al}$ respecto se ha comentado: «Los centros históricos, la parte más significativa y emblemática de la ciudad actual, presentan una problemática particular cuya res-

${ }^{14}$ Como ejemplo de esta situación en el archipiélago, en el texto que el Gobierno de Canarias ofrece en su página especializada de patrimonio histórico, al aludir al conjunto histórico de Gáldar ni se menciona su condición de primera capital de Gran Canaria con la dinastía Semidán o ser el ejemplo más evidente de ciudad prehispánica superpuesta de la comunidad autónoma. Vid. Http:// www.gobiernodecanarias.org/cultura/patrimoniocultural/bics/index.html?bic=true\&cod $=7 \overline{5}$, consulta 10 de marzo de 2019, 13 horas. El texto es el siguiente: «El conjunto histórico integra la Plaza de Santiago y una serie de monumentos que se localizan a su alrededor. El edificio más destacado es la iglesia de Santiago de los Caballeros, que comenzó a levantarse en 1778. De la propia plaza son características las araucarias y los laureles de Indias, que crecen junto a la fuente. En un lateral de este bello recinto se encuentran las Casas Consistoriales, de estilo neoclásico, donde se conserva el Pendón de la Ciudad, la colección local de piezas prehispánicas (sic) y una talla de la Virgen de la Vega. Junto al Ayuntamiento está el Teatro Municipal edificado en 1812 (sic). Otro rincón singular es la Placeta de Santa Lucía. Frente a ella se sitúa el Casino, fundado en 1847 y muy próximo se encuentra el Museo Antonio Padrón, en el que se exhibe una muestra permanente de este pintor galdense».

15 López García, Juan Sebastián (1997): «Ciudad histórica, turismo y revaloración emblemática. Paralelismos entre Guadalajara (Jalisco, México) y La Antigua Guatemala (Sacatepéquez, Guatemala)", $4 .{ }^{\circ}$ Coloquio Internacional Ciudades y Turismo, Nuevos Destinos, Universidad de Guadalajara, México, inédito 2012. López García, J.S., op. cit., p. 176. 
puesta tiene que estar articulada en la estructura general de la ciudad y del territorio. El patrimonio histórico y monumental constituye cada vez más una cuestión territorial $\aleph^{16}$. Hay antecedentes canarios en este enfoque desde finales del siglo Xx, cuando el Cabildo de Gran Canaria consideró el patrimonio histórico como una acción estratégica en el estudio Gran Canaria siglo XXI, que hasta el momento no ha tenido una aplicación efectiva, aunque constituye un interesante antecedente, ya que incluía aspectos relativos al reconocimiento de la territorialidad histórica, conservación del patrimonio inmueble, calidad del paisaje urbano de los centros históricos, actividades económicas, gestión, etc. ${ }^{17}$.

A continuación se ofrece una síntesis de las que se consideran son las aportaciones más destacadas de los conjuntos históricos. Todos esos contenidos con conocidos y documentables, pero sorprendentemente cuando consultamos informaciones sobre estos bienes culturales, incluso en ámbitos que se consideran especializados, no siempre aparecen los aspectos más relevantes, faltando los que van más allá de las descripciones o de lo aparente. Por eso, parece conveniente hacer un repaso breve y muy rápido, ofreciendo un resumen de rasgos de identidad que a la vez son elementos aprovechables para considerar a estos núcleos como un recurso turístico.

\section{Fuerteventura y Lanzarote}

Lanzarote tiene en la villa de San Miguel de Teguise su principal conjunto monumental, que además es superpuesta con la antigua Gran Aldea referida por $L e$ Canarien e identificada con Acatife, cabecera de los antiguos majos. Fue capital de la isla hasta el siglo xix y en su rico patrimonio inmueble está el peculiar trazado irregular y su amplio catálogo de arquitectura doméstica, con predominio de las viviendas terreras, junto a destacados -únicos en algunos casos- ejemplos de distintas tipologías: parroquial de tres naves (parroquia matriz de Nuestra Señora de Guadalupe), iglesias conventuales, casa del señor de la isla, antiguo hospital, cilla, castillo de Santa Bárbara de Guanapay, solar de la mareta, etc. Es decir, en ella coin-

${ }^{16}$ López García, J.S., op. cit., 1997, p. 171.

17 Vid. López García, Juan Sebastián y Hernández Socorro, María de los Reyes (1998): «Patrimonio Histórico», en Gran Canaria. Siglo XXI. Diagnóstico de Situación, t. II, Las Palmas de Gran Canaria, pp. 1506-1548. López García, Juan Sebastián y Hernández Socorro, María de los Reyes (2001): «Patrimonio Histórico-Artístico», en Gran Canaria. Siglo XXI. Cultura y Deporte, Las Palmas de Gran Canaria, p. 47. Las Siete acciones estratégicas en el objetivo estratégico "Promover las actividades de revitalización y conservación del patrimonio histórico de Gran Canaria: Reconocer la territorialidad histórica y conservar el patrimonio monumental inmueble, Conservar y enriquecer el patrimonio histórico mueble y los museos, Mejorar y proteger la calidad del paisaje urbano de los centros históricos, Desarrollar la contemporaneidad de los centros históricos, Incorporar actividades económicas nuevas y desarrollar las tradiciones en los centros históricos, Favorecer la residencia en los centros históricos para su dinamización, Mejorar la gestión de los centros históricos y del patrimonio histórico-artístico». 
ciden los más diversos valores inmuebles, territoriales e intangibles (diabletes, rancho de pascua, etc. $)^{18}$.

Fuerteventura tuvo por capital hasta el siglo xix a la villa de Santa María de Betancuria. El nombre de la localidad recuerda a Jean de Bethencourt, iniciador de la europeización de las Canarias a partir de 1402. Su temprana fundación en el período señorial la convirtió en parroquia matriz de la isla y lugar del primer convento de Canarias (1414), donde estuvo san Diego de Alcalá, así como sede del efímero obispado de Fuerteventura (1424-1433). La excatedral de Santa María de Betancuria es la más destacada muestra de arquitectura religiosa insular, donde se venera a san Buenaventura, patrón insular, atesorando notables bienes muebles, actualmente musealizada. La iglesia sobresale en la villa, en un núcleo que nunca llegó a colmatarse, donde las viviendas más antiguas poseen elementos góticos, la mayoría terreras, no faltando unas pocas de planta alta. El complejo conventual franciscano conserva la ermita de San Diego de Alcalá y las ruinas del templo ${ }^{19}$.

\section{Gran Canaria}

Gran Canaria podría ser un prototipo en cuanto a la relación entre sus conjuntos históricos y la historia de la isla, por los valores, representatividad y significación territorial de la mayoría de ellos. Con la finalización de la conquista en 1483, el campamento de Las Palmas que había sido fundado en 1478 se convierte en la nueva capital, centro de la administración de la corona de Castilla en la isla (Cabildo) y Canarias (obispado y real audiencia). Al mismo tiempo pervivieron en su territorio las dos cabeceras prehispánicas: Gáldar, la primera capital de la isla desde Gumidafe y Andamana, y Telde, además primera sede episcopal de Canarias (1351), muy conocidas por los sobrenombres de «ciudad de los guanartemes" y «ciudad de los faicanes", respectivamente. A partir de estas tres matrices grancanarias se completa el esquema con otras ciudades y villas que se corresponden con los núcleos que se desarrollan a lo largo del siglo XVI, segregadas jurisdiccionalmente de las tres primeras: Arucas, Santa María de Guía, Teror y Santa Brígida, con menor significación territorial, pero con un interesante patrimonio cultural con aportes distintos, de tal manera que se ha resaltado que en el ámbito grancanario cada conjunto pre-

18 Vid. López García, Juan Sebastián (1993): «Aproximación a los núcleos y territorialidad históricos de Lanzarote», Anuario de Estudios Atlánticos, n. ${ }^{\circ} 39$, pp. 611-619.

19 Galante Gómez, Francisco José (2017): «La conquista del espacio en los orígenes de la expansión atlántica. Arte y espiritualidad en el cenobio franciscano de Betancuria», Anuario de Estudios Atlánticos, n. ${ }^{\circ}$ 63, pp. 1-25. Vid para ambas islas, López García, Juan Sebastián (1993): "Núcleos antiguos de Fuerteventura y Lanzarote: análisis histórico, territorial y artístico», en VJornadas de Estudios sobre Fuerteventura y Lanzarote, tomo I, Puerto del Rosario, pp. 308-327. López García, Juan Sebastián (1995): «Patrimonio Histórico: Monumentos y Conjuntos de Lanzarote y Fuerteventura», en VI Jornadas de Estudios sobre Lanzarote y Fuerteventura, Arrecife, pp. 465-497. 
senta un perfil muy diferente de los demás y que los aportes de cada uno se completan con los restantes.

Los centros históricos de Gran Canaria, más allá de los perímetros de sus conjuntos, ofrecen una variada muestra de itinerarios culturales y de patrimonio diverso. Las Palmas de Gran Canaria, con la singular catedral de Santa Ana y una secuencia completa e ininterrumpida de arquitectura desde el gótico hasta las muestras contemporáneas (eclecticismo, modernismo, racionalismo...), más que ninguna otra ciudad isleña. Gáldar, uno de los paradigmas en Canarias del patrimonio, como núcleo representativo de ciudad superpuesta al contar en su centro histórico con una parte de su núcleo canario antiguo, visitable en el Museo y Parque Arqueológico Cueva Pintada, más el conjunto neoclásico de la plaza, consistorio (con drago centenario) y templo de Santiago (primer edificio de ese estilo en el archipiélago) o el arte contemporáneo de Antonio Padrón. Telde, primera sede episcopal de las islas, con su particular vertebración trinuclear de San Juan, San Francisco (Santa María de la Antigua) y San Gregorio (Los Llanos de Jaraquemada), con el especial contraste de los dos primeros barrios entre las casas de la aristocracia y el entorno del convento franciscano y la conexión americana y flamenca en los bienes muebles de su basílica: el Santo Cristo purépecha y el retablo de la Natividad. Santa María de Guía, muy homogénea en su arquitectura de los siglos XVII al XIX con iglesia homónima de fachada barroca y neoclásica que atesora obras de su hijo más ilustre, el escultor José Luján Pérez, con rico patrimonio, destacando en el apartado de lo intangible la Rama y romería de las Marías. Arucas, desarrollada en el xvi por el azúcar, con una arquitectura muy representativa del auge que tuvo la urbe en el siglo XIX con muestras academicistas y eclécticas, donde las labores de su cantería se manifiestan especialmente en la iglesia neogótica de San Juan Bautista, obra cumbre de la arquitectura religiosa canaria del siglo xx. Santa Brígida, villa cabecera de La Vega, con su recoleto casco histórico, donde la parroquia es el emblema de su antigua extensa jurisdicción. Sin significación territorial, Barranco Hondo de Abajo (Gáldar) es representativo del peculiar hábitat en casacueva, tan extendido en Gran Canaria, de origen prehispánico y que en este pago galdense es heredero de uno de los guairatos, el de Artevirgo.

\section{Tenerife}

Tenerife es una isla compleja a la hora de establecer un discurso de jerarquización de sus conjuntos históricos, con la territorialidad histórica y los valores patrimoniales, en una parte por el número de conjuntos históricos declarados y por otra por los cambios de hegemonía de sus núcleos en su proceso de formación histórica. Si se parte desde el momento anterior a la conquista se ofrece el panorama de una isla dividida en menceyatos, pero no hay referencia de poblaciones concretas y sus edificios, como sucede en Gran Canaria con Gáldar. De ahí que la herencia guanche en el territorio sean nombres zonales que pasaron de los menceyatos a los beneficios eclesiásticos más antiguos; mientras unos se asentaron y permanecieron dando nombre a nuevos núcleos, otros desaparecieron, de ahí que el panorama 
resultante sea dispar ${ }^{20}$. Los dos lugares que protagonizan la transición del mundo guanche al hispano están declarados conjuntos históricos, ya que si bien San Cristóbal de La Laguna se funda expresamente para ser capital de la isla en 1497 (para otros en 1496), hay que considerar el protagonismo inicial tras la conquista de Santiago del Realejo de Taoro o Realejo Alto. Al decir de Viera y Clavijo, el beneficio de Taoro es la parroquia más antigua de la isla, contrastando con la opinión de Rodríguez Moure, que considera que es la Concepción lagunera. Sin lugar a dudas lugares protagónicos del territorio tinerfeño y ambos conjuntos históricos que reflejan uno la conexión con el pasado guanche y otro el nuevo orden que se establece en la isla con la incorporación a la corona de Castilla. San Cristóbal de La Laguna tiene el reconocimiento o marca de calidad de ser Patrimonio de la Humanidad, con un trazado singular de tendencia regular estructurado por calles y plazas que tiene en la Carrera el eje que comunica los espacios públicos del núcleo fundacional (plaza de la Concepción) con el «nuevo centro» de su ensanche renacentista del siglo XvI (plaza del Adelantado), pasando por los Remedios. Ciudad que ofrece un catálogo magnífico de arquitectura, especialmente de los siglos XVI al XVIII, con portadas en cantería que caracterizan cada una de esas centurias, en las que se integran viviendas tan singulares como los «palacios» de Nava y del Valle Salazar (actual Obispado). Aporta mucho en la arquitectura religiosa con el templo matriz de la Concepción con una secuencia estilística de varios momentos, la catedral de los Remedios (siglos XIX-XXI), sus conventos de clausura de monacato femenino, iglesias conventuales de órdenes masculinas, ermitas, capillas de cruces, etc., y otros tipos arquitectónicos tales como hospitales, pósito, etc., junto con la arquitectura institucional (antiguo Cabildo, casa del Corregidor y de los Capitanes Generales). Arraigada en el imaginario tinerfeńo, especialmente por la antigua Universidad Literaria de San Fernando, hoy Universidad de La Laguna. Rica en tradiciones, que tiene sus mejores ejemplos en la Semana Santa y las fiestas del Cristo ${ }^{21}$. La Orotava es otro de los ejemplos notables de patrimonio monumental de las islas, que refleja los cambios que se producen en el territorio tinerfeño en el siglo Xvi, al convertirse en el centro del valle de Taoro, al que le dio nombre. Sus ejemplos de arquitectura doméstica son relevantes, así como en otros tipos como la religiosa, utilitaria, jardines, etc., destacando sobre todo el templo de la Concepción, ejemplar más sobresaliente del Barroco de las islas. En su casco histórico se suceden diferentes momentos artísti-

20 Vid. López García, Juan Sebastián (2015): «Territorio y sistema urbano en Tenerife en los siglos Xv y xvi», en Homenaje a la profesora Constanza Negrín Delgado, San Cristóbal de La Laguna, pp. 367-386.

21 Aunque en mayor o menor medida muchos conjuntos históricos cuentan con guías especializadas, La Laguna es un buen ejemplo, a continuación se ofrecen cronológicamente algunos títulos: Cioranescu, Alejandro (1965): La Laguna. Guia histórica y monumental, La Laguna; Fernández Matrán, Miguel Ángel (dir.) (1996): Una ciudad por descubrir. Guia del Centro Histórico de San Cristóbal de La Laguna, Santa Cruz de Tenerife; Marqués Barceló, Carlos y Martín GarCÍA, Aurora (2002): Arquitectura de La Laguna 1496-2002, La Laguna; MarquÉs Barceló, Carlos y Martín García, Aurora (2006): Arquitectura de La Laguna 1496-2005, La Laguna; Balbuena Castellano, Luis (2010): Guía matemática de San Cristóbal de La Laguna, Santa Cruz de Tenerife. 
cos, siendo un paradigma del arte efímero en la celebración de la Octava del Corpus y sus célebres alfombras de tierras de colores. El patrimonio vegetal también está presente en nuestros centros históricos, como sucede en Icod de los Vinos y su drago milenario, además con un hermoso casco histórico y relevantes ejemplos con sus tesoros artísticos, donde destaca el templo de San Marcos y en especial su cruz habanera de filigrana de plata. Un grupo de localidades conservan magníficos centros históricos con muestras arquitectónicas y artísticas notables desde el siglo XVI al XVIII, entre los que destaca Garachico y su posición portuaria, heredera territorial de San Pedro de Daute. Marítima también es Puerto de la Cruz, salida al mar del valle de La Orotava. Con sus aportes particulares, que ahora sería largo de detallar como en las anteriores por el elevando número de conjuntos tinerfeños, se podrían citar, por orden de fecha de declaración: Tacoronte, Tegueste, San Juan de la Rambla, Güímar, Los Silos, villa de Arico, Buenavista del Norte, Realejo Bajo, Arico el Nuevo, Arona, Guía de Isora y San Miguel de Abona. Santa Cruz de Santiago de Tenerife, aunque fundada en la época de la conquista, se desarrolla posteriormente y manifiesta en su trazado y arquitectura, con lo conservado, que fue una importante plaza militar y un puerto internacional destacado, con espacios de los siglos XVIII y XIX que reflejan estas relaciones con otros países en sus artísticos monumentos públicos (Triunfo de la Candelaria, fuente de la Plaza Weyler). De la centuria decimonónica conserva bienes de su época como capital de la entonces provincia de Canarias y como patrimonio inmaterial es de obligada referencia su carnaval. Por último, de la importancia de los núcleos rurales ya se adelantó Adrián Alemán de Armas con sus estudios a partir de los ańos setenta del siglo pasado, declarados están los de Masca (Buenavista del Norte), Icor (Arico), Casas Altas, en Jama (Arona y Vilaflor) y Chirche con Aripe (Guía de Isora) ${ }^{22}$.

\section{La Palma}

La isla de San Miguel de La Palma cuenta con dos conjuntos históricos, que son muy representativos de la isla y de su sistema urbano configurado después de la conquista. Por una parte la nueva capital de la isla y por otro, la población que primero despuntó en el interior al nordeste. Santa Cruz de La Palma (BIC 1975) es el conjunto más importante de su isla y muy valioso para el archipiélago, por lo tangible y lo intangible, ciudad marítima que fue sede del Juzgado de Indias, que concentra en su triangular plaza mayor los ejemplos más representativos de la arquitectura renacentista en Canarias, atesorada por el arte flamenco de sus pinturas y esculturas, escenario de una fiesta única y singular como es la Bajada lustral de la Virgen

22 Vid. Alemán de Armas, Adrián (1975): El caserio de Masca: aproximación a la arquitectura popular canaria, Santa Cruz de Tenerife. Alemán de Armas, A. (1982): «El caserío de Icor: una meditación", en Homenaje a Alfonso Trujillo Rodríguez, t. I, Aula de Cultura de Tenerife, Cabildo de Tenerife, Santa Cruz de Tenerife, pp. 7-45. 
de las Nieves, con su perfil barroco inicial enriquecido en siglos posteriores. San Andrés (BIC 2015), en San Andrés y Sauces, es un núcleo que quedó «congelado» en el siglo XVI, siendo un modelo escueto de una villa que surgió después de la conquista con el cercano ingenio azucarero de Los Sauces ${ }^{23}$, el que fue cantón awarita de Adeyahamen. La villa refleja un trazado bajo el signo de la religiosidad, con el emplazamiento de un calvario en su entrada principal acompañado de la ermita de San Sebastián (protector contra las epidemias), mientras la posición central es ocupada por la iglesia y plaza del apóstol, con ermita de la Virgen del Pilar en su calle principal, completándose el esquema devocional con el desaparecido convento franciscano en las afueras.

\section{CONCLUSIONES}

Una buena parte del legado de Canarias se encuentra en sus conjuntos y centros históricos, poseedores del patrimonio urbano, arquitectónico, artístico, documental, intangible, etc., más representativo de las islas; además, algunos de ellos han sido protagonistas de la construcción histórica del territorio isleño ${ }^{24}$. El reconocimiento oficial del primer bien conjunto histórico fue en 1973, pero la trayectoria ha sido desigual a lo largo de estos años hasta 2018, teniendo como rasgos principales la temprana consolidación de los grancanarios y la más tardía y amplia de los tinerfeños. En este período a los núcleos de arquitectura y arte eruditos, urbanos, se han sumado los de carácter popular, rurales, incluso con la declaración de uno de los primeros como Patrimonio de la Humanidad, San Cristóbal de La Laguna, y la inclusión de uno de los segundos en el perímetro de la candidatura española Unesco en 2018, Barranco Hondo de Abajo (Gáldar), vinculado al paisaje cultural de Risco Caído (perímetro del conjunto histórico integrado en la propuesta compartido con Agaete, Artenara y Tejeda).

La mayoría de los conjuntos históricos tienen emplazamiento interior y alejados de las zonas turísticas; sin embargo, en las últimas décadas se han consolidado como un recurso importante para ofrecer a los viajeros. Este factor los ha dinamizado en lo económico, especialmente en lugares que han apostado por ofrecer mejoras con un patrimonio «visitable» y ampliar su oferta cultural ${ }^{25}$. Sin duda, la situación ha mejorado notablemente si se le compara con las presiones inmobiliarias de finales del siglo $\mathrm{xx}$, aunque persiste la vulnerabilidad en algunos de los campos. En 1998 se denunciaban los efectos de esta especulación que tanto afectó a los bienes inmuebles canarios: «Las presiones y especulaciones sobre los centros históricos

23 Vid López García, Juan Sebastián (1992): «Núcleos y territorialidad históricos de San Miguel de La Palma», Anuario de Estudios Atlánticos, número 38, pp. 503-523.

${ }_{24}$ Associazione Mecenate 90: Léxico dei beni culturali, Roma, 1994, Vid. «Costruzione storica" por Rossi DoRIA, B., pp. 162 y 163.

${ }^{25}$ López García, Juan Sebastián (2003): «Patrimonio cultural y turismo. Manejo, relaciones y repercusiones en Canarias», Patrimonio Cultural y Turismo, cuaderno n. ${ }^{\circ}$ 6, pp. 163-171. 
son constantes, asistiéndose en los ámbitos urbanos a convertir en suelo construible muchos de los espacios ocupados por edificaciones anteriores, con una consecuente sustitución arquitectónica que se apoya en la falta de medidas elementales para proteger a los conjuntos ${ }^{26}$.

En suma, los conjuntos históricos canarios ofrecen aportaciones culturales muy distintas y de tipo variado, en el binomio identidad y patrimonio diverso, conjunción adecuada que lo convierte en un recurso útil de destinos concretos y de itinerarios. Las experiencias de su trayectoria desde 1973 a 2018 deben convertir estos conjuntos históricos en un enclave de la identidad heredada con la conservación y promoción de sus bienes patrimoniales, de encuentro y difusión de los valores culturales, y de promoción de su contemporaneidad como lugares dinámicos y vivos: «El desencuentro entre el pasado y el porvenir se produce cuando el presente renuncie a escribir, tan respetuoso como decididamente, su propio capítulo ${ }^{27}$. El patrimonio de estos bienes canarios es una ruta para interpretar la historia más profunda, y si Celso Martín de Guzmán en 1976 hablaba de «la raíz de la isla», podemos apropiarnos de su idea más de cuarenta años después y decir que en los conjuntos históricos están las raíces culturales de nuestro archipiélago ${ }^{28}$.

Recibido: 20-03-2019. Aceptado: 11-04-2019

${ }^{26}$ López García, Juan Sebastián (1998): «Los Centros Históricos Canarios y el horizonte del 2000", en IV Congreso Internacional de Rehabilitación del Patrimonio Arquitectónico y Edificación. Cuba'98, La Laguna, p. 364.

${ }_{27}$ Pérez Escolano, Víctor (1994): «Encuentros y desencuentros entre pasado y porvenir. Un apunte», Cuadernos. Patrimonio y Ciudad. Reflexión sobre Centros Históricos, v, Córdoba, p. 120.

${ }^{28}$ Martín de Guzmán, Celso (1976): «La raíz de la isla», en Programa de las Fiestas Mayores de Santiago, Gáldar, s.p. 


\section{ANEXO I}

Conjuntos históricos de Canarias. Orden CRONOlÓgico Declaraciones realizadas por el Gobierno de España

- Las Raíces, La Esperanza, El Rosario: D. 4395/1964, de 23 de diciembre.

- Barrio de Vegueta, Las Palmas de Gran Canaria: D. 881/1973, de 5 de abril.

- Santa Cruz de La Palma: D. 942/1975, de 10 de abril.

- La Orotava: R.D. 3302/1976, de 10 de diciembre.

- Arucas: R.D. 3303/1976, de 10 de diciembre.

- Betancuria: R.D. 3086/1978, de 10 de noviembre.

- Teror: R.D. 690/1979, de 13 de febrero.

- Castillo de San Gabriel, su camino de acceso y su puente levadizo: R.D. 1781/1979, de 16 de junio.

- Teguise: R.D. 3035/1980, de 21 de noviembre.

- Tacoronte: R.D. 3047/1980, de 12 de diciembre.

- San Juan y San Francisco, Telde: R.D. 1121/1981 de 6 de marzo.

- Plaza de Santiago, Gáldar, Gáldar: R.D. 1048/1981 de 13 de marzo.

- Santa María de Guía: R.D. 2720/1982, de 27 de agosto.

\section{Declaraciones realizadas por el Gobierno de Canarias}

- San Cristóbal de La Laguna: D. 602/1985, de 20 de diciembre.

- Tegueste: D. 158/1986, de 24 de octubre.

- Barrio de Triana, Las Palmas de Gran Canaria (1990): O. de 2 de julio de 1993, por la que se da publicidad al Acuerdo de Gobierno de 19 de noviembre de 1990.

- San Juan de la Rambla (1990): O. de 2 de julio de 1993, de publicidad de Acuerdo del Gobierno de Canarias de 28 de noviembre de 1990.

- Barranco Hondo de Abajo, Gáldar: D. 258/1993, de 24 de septiembre.

- Garachico: D.10/1994 de 11 de febrero.

- Casas de La Mayordomía y ermita de San Antonio Abad, Tamaraceite, Las Palmas de Gran Canaria: D. 152/1995, de 9 de junio.

- Güímar: D. 180/2001 de 17 de septiembre.

- Casas Altas, en Jama, Arona y Vilaflor: D. 125/2004, de 7 de septiembre.

- Masca, Buenavista del Norte: D. 126/2004, de 7 de septiembre.

- Los Silos: D. 137/2004 de 29 de septiembre.

- Icod de los Vinos: D. 142/2004, de 5 de octubre.

- Villa de Arico, Arico: D. 71/2005, de 4 de marzo.

- Buenavista del Norte: D. 21/2005, de 22 de febrero.

- Icor, Arico: D. 76/2005, de 17 de mayo.

- Taucho (Adeje): D. 94/2005, de 24 de mayo.

- Realejo Bajo, Los Realejos: D. 220/2005 de 1 de diciembre. 


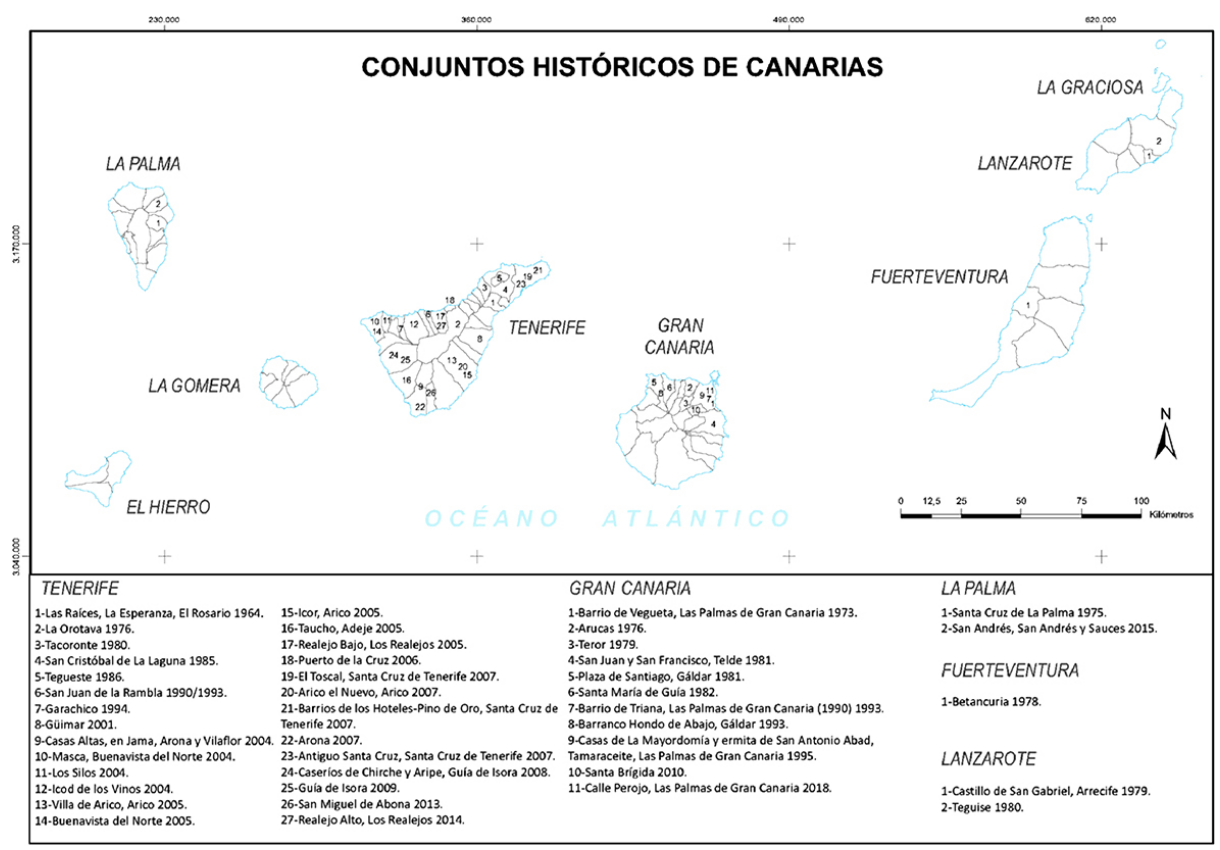

- Puerto de la Cruz: D. 65/2006 de 23 de mayo.

- El Toscal, Santa Cruz de Tenerife: D. 2/2007, de 16 de enero.

- Arico el Nuevo, Arico: D. 14/2007, de 5 de febrero.

- Barrios de los Hoteles-Pino de Oro, Santa Cruz de Tenerife: D. 67/2007, de 2 de abril. Modificada la delimitación por D. 111/2014, de 28 de noviembre.

- Arona: D. 70/2007, de 2 de abril.

- Antiguo Santa Cruz, Santa Cruz de Tenerife (2007): D. 299/2007, de 31 de julio. Modificada la delimitación por D. 131/2014, de 29 de diciembre.

- Caseríos de Chirche y Aripe, Guía de Isora: D.223/2008 de 18 de noviembre.

- Guía de Isora: D. 27/2009, de 10 de marzo.

- Santa Brígida: D. 252/2010, de 27 de agosto.

- San Miguel de Abona: D. 51/2013, de 16 de mayo.

- Realejo Alto, Los Realejos: D. 45/2014, de 22 de mayo.

- San Andrés, San Andrés y Sauces: D. 18/2015, de 26 de febrero.

- Calle Perojo, Las Palmas de Gran Canaria, D. 46/2018, de 16 de abril. 


\section{ANEXO II}

Conjuntos históricos DE CANARIAS. RELACIÓN POR ISLAS

Gran Canaria

- Barrio de Vegueta, Las Palmas de Gran Canaria: D. 881/1973, de 5 de abril.

- Arucas: R.D. 3303/1976, de 10 de diciembre.

- Teror: R.D. 690/1979, de 13 de febrero.

- San Juan y San Francisco, Telde: R.D. 1121/1981 de 6 de marzo.

- Plaza de Santiago, Gáldar, Gáldar: R.D. 1048/1981 de 13 de marzo.

- Santa María de Guía: R.D. 2720/1982, de 27 de agosto.

- Barrio de Triana, Las Palmas de Gran Canaria (1990): O. de 2 de julio de 1993, por la que se da publicidad al Acuerdo de Gobierno de 19 de noviembre de 1990.

- Barranco Hondo de Abajo, Gáldar: D. 258/1993, de 24 de septiembre.

- Casas de La Mayordomía y ermita de San Antonio Abad, Tamaraceite, Las Palmas de Gran Canaria: D. 152/1995, de 9 de junio.

- Santa Brígida: D. 252/2010, de 27 de agosto.

- Calle Perojo, Las Palmas de Gran Canaria, D. 46/2018, de 16 de abril.

\section{Fuerteventura}

- Betancuria: R.D. 3086/1978, de 10 de noviembre.

\section{Lanzarote}

- Castillo de San Gabriel, su camino de acceso y su puente levadizo: R.D. 1781/1979, de 16 de junio.

- Teguise: R.D. 3035/1980, de 21 de noviembre.

\section{La Palma}

- Santa Cruz de La Palma: D. 942/1975, de 10 de abril.

- San Andrés, San Andrés y Sauces: D. 18/2015, de 26 de febrero.

\section{Tenerife}

- Las Raíces, La Esperanza, El Rosario: D. 4395/1964, de 23 de diciembre.

- La Orotava: R.D. 3302/1976, de 10 de diciembre.

- Tacoronte: R.D. 3047/1980, de 12 de diciembre.

- San Cristóbal de La Laguna: D. 602/1985, de 20 de diciembre. 


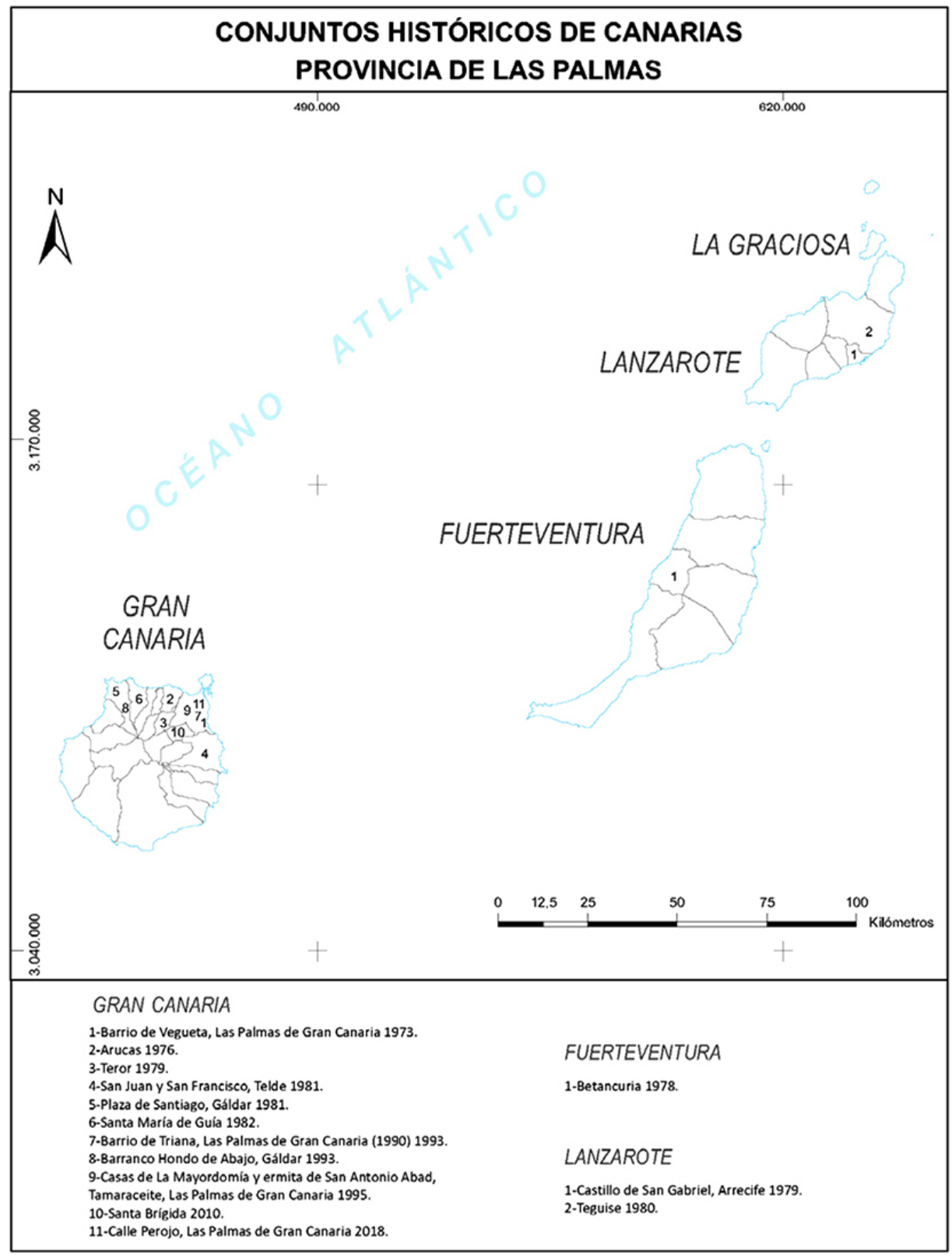




\section{CONJUNTOS HISTÓRICOS DE CANARIAS \\ PROVINCIA DE SANTA CRUZ DE TENERIFE}

230.000

360.000

\section{LAPALMA}

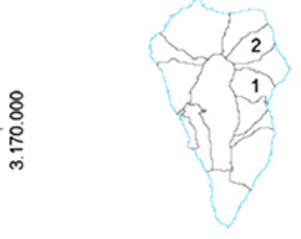

LA GOMERA
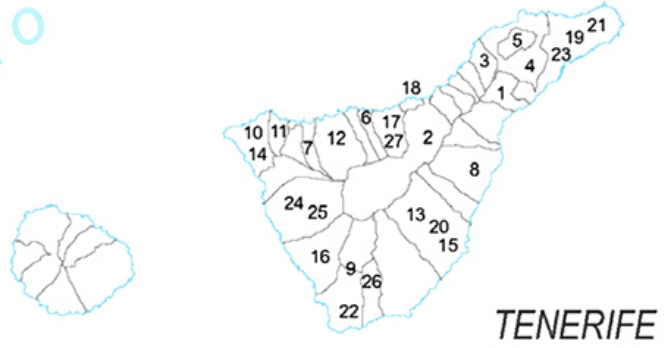

EL HIERRO

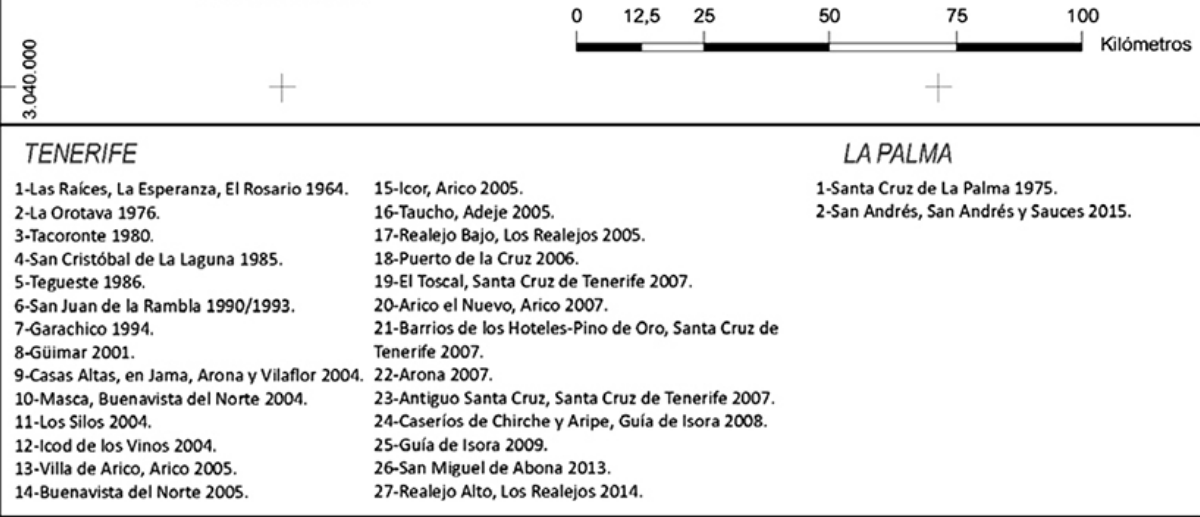


- Tegueste: D. 158/1986, de 24 de octubre.

- San Juan de la Rambla (1990): O. de 2 de julio de 1993, de publicidad de Acuerdo del Gobierno de Canarias de 28 de noviembre de 1990.

- Garachico: D.10/1994 de 11 de febrero.

- Güímar: D. 180/2001 de 17 de septiembre.

- Casas Altas, en Jama, Arona y Vilaflor: D. 125/2004, de 7 de septiembre.

- Masca, Buenavista del Norte: D. 126/2004, de 7 de septiembre.

- Los Silos: D. 137/2004 de 29 de septiembre.

- Icod de los Vinos: D. 142/2004, de 5 de octubre.

- Villa de Arico, Arico: D. 71/2005, de 4 de marzo.

- Buenavista del Norte: D. 21/2005, de 22 de febrero.

- Icor, Arico: D. 76/2005, de 17 de mayo.

- Taucho (Adeje): D. 94/2005, de 24 de mayo.

- Realejo Bajo, Los Realejos: D. 220/2005 de 1 de diciembre.

- Puerto de la Cruz: D. 65/2006 de 23 de mayo.

- El Toscal, Santa Cruz de Tenerife: D. 2/2007, de 16 de enero.

- Arico el Nuevo, Arico: D. 14/2007, de 5 de febrero.

- Barrios de los Hoteles-Pino de Oro, Santa Cruz de Tenerife: D. 67/2007, de 2 de abril. Modificada la delimitación por D. 111/2014, de 28 de noviembre.

- Arona: D. 70/2007, de 2 de abril.

- Antiguo Santa Cruz, Santa Cruz de Tenerife (2007): D. 299/2007, de 31 de julio. Modificada la delimitación por D. 131/2014, de 29 de diciembre.

- Caseríos de Chirche y Aripe, Guía de Isora: D.223/2008 de 18 de noviembre.

- Guía de Isora: D. 27/2009, de 10 de marzo.

- San Miguel de Abona: D. 51/2013, de 16 de mayo.

- Realejo Alto, Los Realejos: D. 45/2014, de 22 de mayo.

Nota: No consideramos para este estudio, aunque lo sean legalmente, por ser dudosa la categoría para ellos: Las Raíces, castillo de San Gabriel y la Mayordomía de Tamaraceite.

Fuentes: Gobierno de Canarias. Consejería de Turismo, Cultura y Deportes. Relación de Bienes de Interés Cultural. Cabildo de Gran Canaria, Servicio de Patrimonio Histórico. Base cartográfica Grafcan: elaboración propia, con asesoramiento técnico de Antonio Bueno García. 
